\title{
A singular series average and Goldbach numbers in short intervals
}

by

\author{
A. Languasco (Genova)
}

1. Introduction. The Goldbach conjecture asserts that every even integer greater than two can be written as a sum of two primes. We will call the even numbers satisfying Goldbach's conjecture G-numbers.

Let $X$ be a sufficiently large parameter. Our aim here is to study Gnumbers in short intervals. The first unconditional result of this kind was obtained by Ramachandra [8] who proved that there is a positive proportion of G-numbers in every interval $[X, X+H]$ for $H \geq X^{7 / 72+\varepsilon}$. The best known result is now $H \geq X^{0.535 / 20+\varepsilon}$ (see Baker-Harman-Pintz [1]).

From a conditional viewpoint, we recall that, assuming the Riemann Hypothesis $(\mathrm{RH})$, Goldston [3] proved that there is a positive proportion of G-numbers in every interval of length $H \gg \log ^{2} X$. The existence of G-numbers in such intervals was established independently by Kátai [5] and Montgomery-Vaughan [7]. Assuming further Montgomery's pair correlation conjecture (MC), Goldston [3] was also able to restrict the length of the interval to $H \geq \log ^{1+\varepsilon} X$, for every fixed $\varepsilon>0$. If we only ask about the existence of a G-number in short interval, we recall that Goldston [3] (see also Languasco [6]) proved, again under $\mathrm{RH}$ and $\mathrm{MC}$, that in every interval of length $H \gg \log X$ there exists a G-number. The loss of a factor $(\log X)^{\varepsilon}$ in the result on the positive proportion compared with the one on the existence is due to some problems connected with averages of the singular series of the Goldbach problem.

We introduce the following notation. Let

$$
R(n)=\sum_{p_{1}+p_{2}=n} \log p_{1} \log p_{2}
$$

be the weighted counting function of Goldbach numbers and

1991 Mathematics Subject Classification: Primary 11P32.

Research supported by the CNR grant no. 201.01.123. 


$$
\mathfrak{S}(n)= \begin{cases}2 \prod_{p>2}\left(1-\frac{1}{(p-1)^{2}}\right) \prod_{\substack{p \mid n \\ p>2}}\left(\frac{p-1}{p-2}\right) & \text { if } n \text { is even } \\ 0 & \text { if } n \text { is odd }\end{cases}
$$

the singular series of the Goldbach problem.

The main result of this paper is

Theorem. Let $\nu \in \mathbb{R}, \nu \geq 1$, be fixed. Then

$$
\sum_{n \leq X} \mathfrak{S}(n)^{\nu}=c_{1} X+c_{2}(\log X)^{\nu}+O\left((\log X)^{\nu-1 / 3}\right),
$$

where $c_{1}, c_{2}$ and the implicit constant depend on $\nu$.

The exponent $\nu-1 / 3$ in the error term comes from an appeal to Vinogradov's estimate for exponential sums. In particular, we will use the Walfisz [10] estimate

$$
\sum_{n \leq X} \frac{1}{n} P\left(\frac{X}{n}\right) \ll(\log X)^{2 / 3},
$$

where $P(u)=u-[u]-1 / 2$.

We remark that the proof of the Theorem is based on the argument in Friedlander-Goldston [2]. We also remark that the case $\nu=1$ of our Theorem is proved in Friedlander-Goldston [2] (see (1.13) there), and that the case $\nu=2$ is proved in Languasco [6].

The link between the average of the singular series and the positive proportion of G-numbers is given by the following

Corollary. Let $\varepsilon>0$ be fixed. Assume, for $X$ sufficiently large, that

$$
\sum_{n=0}^{H}(H-n) R(n+X) \gg H^{2} X \quad \text { for some }(\log X)^{2 / 3+\varepsilon} \leq H \leq X .
$$

Then there are $\gg_{\varepsilon} H$ G-numbers in the interval $[X, X+H]$.

We remark that $(2)$ is essentially the condition that all papers concerning the existence of G-numbers in short intervals check. From the proof of the Corollary it is easy to note that we could assume $\sum_{n=X}^{X+H} R(n) \gg H X$ instead of (2).

Inserting the Corollary in the body of the proof of Languasco [6], we can fill the gap between the result on the existence and on the positive proportion of G-numbers, i.e., we can prove, assuming $\mathrm{RH}$ and a weak form of MC, that every interval of length $H \gg \log X$ contains a positive proportion of G-numbers. Moreover, inserting our Corollary at the end of the proof of the Friedlander-Goldston [2] result, we are able to obtain, under the assumption of $\mathrm{RH}, \mathrm{MC}$ and the Elliott-Halberstam conjecture of level 
$Q=X \exp \left(-(\log X)^{2 / 3}\right)$, that every interval of length $H \gg(\log X)^{2 / 3+\varepsilon}$ contains a positive proportion of G-numbers. This seems to be the limit of the method.

We finally recall that Friedlander and Goldston [2] were able to prove the existence of G-numbers in intervals shorter than $(\log X)^{2 / 3+\varepsilon}$ by assuming a larger level $Q$ in the Elliott-Halberstam conjecture.

2. A generalization of the Walfisz estimate. Let $\nu \in \mathbb{R}, \nu \geq 1$, and $s=\sigma+i t$. We define the general divisor function $d_{\nu}(n)$ by

$$
\sum_{n=1}^{\infty} d_{\nu}(n) n^{-s}=\zeta(s)^{\nu} \quad \text { for } \sigma>1 .
$$

From the definition it is clear that $d_{\nu}(n)$ is a multiplicative function which extends the well known function $d_{k}(n)$, where $k$ is an integer. Under this notation, the usual divisor function $d(n)$ is denoted by $d_{2}(n)$. Our result is

Lemma. Let $\nu \in \mathbb{R}, \nu \geq 1$. Then

$$
\sum_{n \leq X} \frac{d_{\nu}(n)}{n} P\left(\frac{X}{n}\right) \ll_{\nu}(\log X)^{\nu-1 / 3} .
$$

We remark that for $\nu \in \mathbb{N}$ the above Lemma is stated at the end of Section 2 of Friedlander-Goldston [2].

Proof. First of all suppose that $\nu$ is an integer. The case $\nu=1$ is exactly (1). If $\nu=2$, using the hyperbola principle and (1) we get

(4) $\sum_{n \leq X} \frac{d(n)}{n} P\left(\frac{X}{n}\right) \ll \sum_{m \leq X} \frac{1}{m} \sum_{n \leq X / m} \frac{1}{n} P\left(\frac{X / m}{n}\right) \ll(\log X)^{2 / 3} \sum_{m \leq X} \frac{1}{m}$

$$
\ll(\log X)^{5 / 3} \text {. }
$$

Suppose now that $\nu \geq 3$. Then

$$
d_{\nu}(n)=\left(d_{\nu-1} * \mathbf{1}\right)(n)
$$

and so, using the definition of convolution, we get

$$
\sum_{n \leq X} \frac{d_{\nu}(n)}{n} P\left(\frac{X}{n}\right) \ll \sum_{m \leq X} \frac{1}{m} \sum_{n \leq X / m} \frac{d_{\nu-1}(n)}{n} P\left(\frac{X / m}{n}\right) .
$$

Hence, arguing by induction and using (4)-(5), we obtain

$$
\sum_{n \leq X} \frac{d_{\nu}(n)}{n} P\left(\frac{X}{n}\right) \ll_{\nu}(\log X)^{\nu-1 / 3} \quad \text { for } \nu \in \mathbb{N} .
$$

Now take $\nu \in \mathbb{R} \backslash \mathbb{N}, \nu>1$. It is easy to see that $d_{\nu}(n)=\left(d_{[\nu]} * d_{\{\nu\}}\right)(n)$, where $[x]$ and $\{x\}$ are, respectively, the integer part and the fractional part 
of $x$. Using (6), we can write

$$
\begin{aligned}
\sum_{n \leq X} \frac{d_{\nu}(n)}{n} P\left(\frac{X}{n}\right) & \ll \sum_{m \leq X} \frac{d_{\{\nu\}}(m)}{m} \sum_{n \leq X / m} \frac{d_{[\nu]}(n)}{n} P\left(\frac{X / m}{n}\right) \\
& \ll_{\nu}(\log X)^{[\nu]-1 / 3} \sum_{m \leq X} \frac{d_{\{\nu\}}(m)}{m} .
\end{aligned}
$$

Using

$$
\sum_{m \leq X} d_{\{\nu\}}(m) \ll_{\nu} X(\log X)^{\{\nu\}-1},
$$

which follows from (II.5.32) of Tenenbaum [9], we obtain, by partial summation,

$$
\sum_{m \leq X} \frac{d_{\{\nu\}}(m)}{m} \ll(\log X)^{\{\nu\}} .
$$

From (7) and (8) we have the Lemma.

3. Proof of the Theorem. We follow the argument in FriedlanderGoldston [2]. Let

$$
\mathfrak{S}=\prod_{p>2}\left(1-\frac{1}{(p-1)^{2}}\right)
$$

We have

$$
\begin{aligned}
\sum_{n \leq X} \mathfrak{S}(n)^{\nu} & =(2 \mathfrak{S})^{\nu} \sum_{2 n \leq X} \prod_{\substack{p \mid n \\
p>2}}\left(1+\frac{1}{p-2}\right)^{\nu} \\
& =(2 \mathfrak{S})^{\nu} \sum_{2 n \leq X} \prod_{\substack{p \mid n \\
p>2}}\left(1+\left(\left(1+\frac{1}{p-2}\right)^{\nu}-1\right)\right) \\
& =(2 \mathfrak{S})^{\nu} \sum_{n \leq X / 2} \sum_{j \mid n} f_{\nu}(j),
\end{aligned}
$$

where

$$
f_{\nu}(j)= \begin{cases}\mu^{2}(j) \prod_{p \mid j}\left(\left(1+\frac{1}{p-2}\right)^{\nu}-1\right) & \text { if } j \text { is odd } \\ 0 & \text { if } j \text { is even. }\end{cases}
$$

Then, changing the order of summation in (9), we obtain 


$$
\begin{aligned}
\sum_{n \leq X} \mathfrak{S}(n)^{\nu}= & (2 \mathfrak{S})^{\nu} \sum_{j \leq X / 2} f_{\nu}(j)\left[\frac{X}{2 j}\right] \\
= & \frac{(2 \mathfrak{S})^{\nu}}{2} X \sum_{j=1}^{\infty} \frac{f_{\nu}(j)}{j}-\frac{(2 \mathfrak{S})^{\nu}}{2} X \sum_{j>X / 2} \frac{f_{\nu}(j)}{j} \\
& -\frac{(2 \mathfrak{S})^{\nu}}{2} \sum_{j \leq X / 2} f_{\nu}(j)-(2 \mathfrak{S})^{\nu} \sum_{j \leq X / 2} f_{\nu}(j) P\left(\frac{X}{2 j}\right),
\end{aligned}
$$

where $P(u)=u-[u]-1 / 2$.

By straightforward computations we get

$$
\begin{aligned}
& \frac{(2 \mathfrak{S})^{\nu}}{2} \sum_{j=1}^{\infty} \frac{f_{\nu}(j)}{j} \\
& =2^{\nu-1} \prod_{p>2}\left(1-\frac{1}{(p-1)^{2}}\right)^{\nu}\left(1+\frac{1}{p}\left(\left(1+\frac{1}{p-2}\right)^{\nu}-1\right)\right)=c_{1}(\nu),
\end{aligned}
$$

since the infinite product is convergent.

Now we will proceed to prove that

$$
\sum_{\nu}:=\sum_{j \leq X / 2} f_{\nu}(j) P\left(\frac{X}{2 j}\right) \ll_{\nu}(\log X)^{\nu-1 / 3} \quad \text { for } \nu \geq 1 .
$$

If $\nu=1$ we have, for $j$ odd,

$$
f_{1}(j)=\mu^{2}(j) \prod_{p \mid j} \frac{1}{p-2}=\frac{\mu^{2}(j)}{\varphi_{2}(j)}
$$

where $\varphi_{2}(p)=p-2$ and $\varphi_{2}$ is extended to square-free numbers by multiplicativity. Hence

$$
\sum_{1}=\sum_{\substack{j \leq X / 2 \\(j, 2)=1}} \frac{\mu^{2}(j)}{\varphi_{2}(j)} P\left(\frac{X}{2 j}\right) \ll(\log X)^{2 / 3},
$$

where the last inequality follows using (2.9)-(2.13) of Friedlander-Goldston [2] and the Lemma.

Let now $\nu>1$. For $j$ odd, we have

$$
f_{\nu}(j)=\mu^{2}(j) \prod_{p \mid j}\left(\left(1+\frac{1}{p-2}\right)^{\nu}-1\right)
$$

and hence, using the Taylor expansion of $(1+1 /(p-2))^{\nu}$, we get 


$$
\begin{aligned}
f_{\nu}(j) & =\mu^{2}(j) \prod_{p \mid j}\left(\sum_{k=0}^{\infty} \frac{\prod_{h=0}^{k}(\nu-h)}{k !(p-2)^{k+1}}\right) \\
& =\mu^{2}(j) \prod_{p \mid j} \frac{\nu}{p-2}\left(1+\sum_{k=1}^{\infty} \frac{\prod_{h=1}^{k}(\nu-h)}{(k+1) !(p-2)^{k}}\right) \\
& =\mu^{2}(j) \frac{d_{\nu}(j)}{\varphi_{2}(j)} \sum_{\delta \mid j} \mu^{2}(\delta) g_{\nu}(\delta),
\end{aligned}
$$

where

$$
g_{\nu}(p)=\sum_{k=1}^{\infty} \frac{\prod_{h=1}^{k}(\nu-h)}{(k+1) !(p-2)^{k}}
$$

and $g_{\nu}$ is extended to square-free numbers by multiplicativity.

We remark that

$$
g_{\nu}(p)=\frac{\nu-1}{p-2} \sum_{k=2}^{\infty} \frac{\prod_{h=2}^{k-1}(\nu-h)}{k !(p-2)^{k-2}} \ll_{\nu} \frac{1}{p-2}
$$

and hence, for $\delta$ square-free, we have $g_{\nu}(\delta) \ll_{\nu}\left(\varphi_{2}(\delta)\right)^{-1}$.

Hence, inserting (14) into $\sum_{\nu}$ and interchanging the order of summation, we obtain

$$
\sum_{\nu} \ll_{\nu} \sum_{\substack{\delta \leq X / 2 \\(\delta, 2)=1}} \frac{\mu^{2}(\delta) d_{\nu}(\delta)}{\left(\varphi_{2}(\delta)\right)^{2}}\left(\sum_{\substack{k \leq X /(2 \delta) \\(k, 2)=1}} \frac{\mu^{2}(k) d_{\nu}(k)}{\varphi_{2}(k)} P\left(\frac{X /(2 \delta)}{k}\right)\right) .
$$

Using the argument in (2.9)-(2.13) of Friedlander-Goldston [2], we can estimate the inner sum by

$$
\sum_{n \leq X} \frac{d_{\nu}(n)}{n} P(X / n)
$$

which is $\ll(\log X)^{\nu-1 / 3}$ by the Lemma. Using this estimate we have

$$
\sum_{\nu} \ll_{\nu}(\log X)^{\nu-1 / 3} \sum_{\substack{\delta \leq X / 2 \\(\delta, 2)=1}} \frac{\mu^{2}(\delta) d_{\nu}(\delta)}{\left(\varphi_{2}(\delta)\right)^{2}} \ll_{\nu}(\log X)^{\nu-1 / 3},
$$

where the last inequality follows from the convergence of the series

$$
\sum_{\substack{\delta=1 \\(\delta, 2)=1}}^{\infty} \frac{\mu^{2}(\delta) d_{\nu}(\delta)}{\left(\varphi_{2}(\delta)\right)^{2}}
$$

Combining (13) and (15) we obtain (12). 
Next, we will show

$$
\sum_{j \leq X / 2} f_{\nu}(j)=c_{2}(\nu)(\log X)^{\nu}+O_{\nu}\left((\log X)^{\nu-1}\right) .
$$

By partial summation this implies

$$
\sum_{j>X / 2} \frac{f_{\nu}(j)}{j} \ll_{\nu} \frac{(\log X)^{\nu-1}}{X} .
$$

Now we prove (16). If $\nu \in \mathbb{N}$ we can argue as in the proof of the Proposition of Languasco [6]. The only difference is that we use the function

$$
H_{\nu}(s)=\sum_{m=1}^{\infty} f_{\nu}(m) m^{-s}=\left(1-\frac{1}{2^{s+1}}\right)^{\nu} \zeta(s+1)^{\nu} G_{\nu}(s),
$$

where

$$
G_{\nu}(s)=\prod_{p>2}\left(1-\frac{1}{p^{s+1}}\right)^{\nu}\left[1+\frac{1}{p^{s}}\left(\left(1+\frac{1}{p-2}\right)^{\nu}-1\right)\right]
$$

converges absolutely and is analytic for $\sigma>-1 / 2$. Moreover, $H_{\nu}(s)(X / 2)^{s} / s$ has a pole of order $\nu+1$ at $s=0$ with residue

$$
c_{2}(\nu)(\log X)^{\nu}+O_{\nu}\left((\log X)^{\nu-1}\right),
$$

and so, using the Perron formula, we find that (16) holds for $\nu \in \mathbb{N}$.

Now let $\nu \in \mathbb{R} \backslash \mathbb{N}, \nu>1$. In this case $H_{\nu}(s)$ has a singularity at $s=0$ that is not a pole, so we cannot use the previous argument.

Let

$$
F_{\nu}(s)=H_{\nu}(s-1)=\left(1-\frac{1}{2^{s}}\right)^{\nu} \zeta(s)^{\nu} G_{\nu}(s-1) .
$$

We apply the Selberg-Delange method to study the contribution of the non-polar singularity at $s=1$ of $F_{\nu}(s)$. By Theorem II.5.3 of Tenenbaum [9] we obtain

$$
S(Y)=\sum_{m \leq Y} m f_{\nu}(m)=k(\nu) Y(\log Y)^{\nu-1}+O_{\nu}\left(Y(\log Y)^{\nu-2}\right),
$$

where $k(\nu)$ and the implicit constant depend on $\nu$. Equation (16) now follows since

$$
\begin{aligned}
\sum_{j \leq X / 2} f_{\nu}(j) & =\int_{1}^{X / 2} \frac{1}{u} d S(u)=\frac{2 S(X / 2)}{X}+\int_{1}^{X / 2} \frac{S(u)}{u^{2}} d u \\
& =\frac{k(\nu)}{\nu}(\log X)^{\nu}+O_{\nu}\left((\log X)^{\nu-1}\right) .
\end{aligned}
$$

The Theorem now follows upon inserting (11)-(12) and (16)-(17) in (10). 
4. Proof of the Corollary. We give a sketch of the proof, which follows the usual pattern (see, e.g., Goldston [3]).

Let $\nu=1+\varepsilon / 2$. Using the Hölder inequality and (2), we obtain

$$
\begin{aligned}
\sum_{\substack{n=X \\
R(n) \geq 1}}^{X+H} 1 & \geq \frac{\left(\sum_{n=0}^{H}(H-n) R(n+X)\right)^{\nu /(\nu-1)}}{\left(\sum_{n=0}^{H}((H-n) R(n+X))^{\nu}\right)^{1 /(\nu-1)}} \\
& \gg \frac{\left(H^{2} X\right)^{\nu /(\nu-1)}}{\left(\sum_{n=0}^{H}((H-n) R(n+X))^{\nu}\right)^{1 /(\nu-1)}} .
\end{aligned}
$$

Since

$$
\sum_{n=0}^{H}((H-n) R(n+X))^{\nu} \ll H^{\nu} \sum_{n=X}^{X+H} R(n)^{\nu}
$$

and, by the well known sieve upper bound (see Halberstam-Richert [4], Theorem 3.11),

$$
R(n) \ll n \mathfrak{S}(n),
$$

we get, using also the Theorem,

$$
\begin{aligned}
\sum_{n=0}^{H}((H-n) R(n+X))^{\nu} & \ll H^{\nu} X^{\nu} \sum_{n=X}^{X+H} \mathfrak{S}(n)^{\nu} \\
& =H^{\nu} X^{\nu}\left(c_{1}(\nu) H+O_{\varepsilon}\left((\log X)^{2 / 3+\varepsilon / 2}\right)\right) \\
& \ll{ }_{\varepsilon} H^{\nu+1} X^{\nu}
\end{aligned}
$$

Hence by (18)-(19) we obtain

$$
\sum_{\substack{n=X \\ R(n) \geq 1}}^{X+H} 1 \gg_{\varepsilon} \frac{\left(H^{2} X\right)^{\nu /(\nu-1)}}{\left(H^{\nu+1} X^{\nu}\right)^{1 /(\nu-1)}}=H
$$

and the Corollary follows.

\section{References}

[1] R. Baker, G. Harman and J. Pintz, The exceptional set for Goldbach's problem in short intervals, in: Proc. of Sieve Methods, Exponential Sums and Their Application in Number Theory, G. R. H. Greaves et al. (eds.), Cambridge Univ. Press, 1996, $1-54$.

[2] J. B. Friedlander and D. A. Goldston, Some singular series averages and the distribution of Goldbach numbers in short intervals, Illinois J. Math. 39 (1995), 158-180.

[3] D. A. Goldston, Linnik's theorem on Goldbach numbers in short intervals, Glasgow Math. J. 32 (1990), 285-297.

[4] H. Halberstam and H.-E. Richert, Sieve Methods, Academic Press, 1974. 
[5] I. Kátai, A remark on a paper of Yu. V. Linnik, Magyar Tud. Akad. Mat. Fiz. Oszt. Közl. 17 (1967), 99-100 (in Hungarian).

[6] A. Languasco, A conditional result on Goldbach numbers in short intervals, Acta Arith., this volume, 93-103.

[7] H. L. Montgomery and R. C. Vaughan, The exceptional set in Goldbach's problem, ibid. 27 (1975), 353-370.

[8] K. Ramachandra, Two remarks in prime number theory, Bull. Soc. Math. France 105 (1977), 433-437.

[9] G. Tenenbaum, Introduction à la théorie analytique et probabiliste des nombres, Publ. Inst. E. Cartan 13, 1990.

[10] A. Walfisz, Weylsche Exponentialsummen in der neueren Zahlentheorie, Deutscher Verlag Wiss., 1963.

Dipartimento di Matematica

Università di Genova

Via Dodecaneso 35

16146 Genova, Italy

E-mail: languasco@dima.unige.it

Received on 14.4.1997

and in revised form on 8.9.1997 